\title{
AUTOMATIC LUMINOSITY OPTIMIZATION OF THE ILC HEAD-ON BEAM DELIVERY SYSTEM*
}

\author{
J. Payet, A. Chancé, O. Napoly, D. Uriot CEA/DSM/DAPNIA/SACM, Saclay, France \\ S. Auclair, EFREI, Paris, France
}

\section{Abstract}

With the local chromaticity correction scheme [1], the luminosity optimisation of the $e+e-$ International Linear Collider (ILC) beam delivery system is challenging. It is a long and complex process and its automation becomes a necessity. It was recently shown that it is possible to employ a simplex minimization method of the beam sizes at the Interaction Point (IP) [2], without adding any other constraint on the beam line parameters. To achieve this goal, we have developed a minimization code which uses analytical computations of the IP beam sizes based on the high order transport coefficients calculated by the codes TRANSPORT [3]. We also use the code TRACEWIN [4] which tracks a particle cloud and minimise the rms beam sizes at the IP to optimise the luminosity, and we compare the results.

\section{BDS OPTICS}

We have studied, for the two energies $250 \mathrm{GeV}$ and $500 \mathrm{GeV}$, the Beam Delivery System (BDS) of a head-on interaction region for the ILC, based on a new scheme for the extraction beam line [5].The parameters are the ILC nominal parameters as listed in Table 1.

The distance $l^{*}$ between the IP and the FD is set to $4.0 \mathrm{~m}$. to keep the final quadrupole away from the solenoid field. The FD quadrupoles are superconducting magnets like the TESLA FD, and in order to extract the spent beam, a $28 \mathrm{~m}$ long electrostatic separator [6] is located as close to the IP as possible. A layout of the $500 \mathrm{GeV}$ version of the FD region is shown on Figure 1.

For the line, we adapt the ILC 2006e beam focusing system [7] to the head-on case. To improve our chromatic correction, a sextupole is added at the middle of the energy collimation section. Figure 2 shows the optical functions of the BDS.

Table 1: Beam parameters

\begin{tabular}{|l|l|l|}
\hline Beam energy $(\mathrm{GeV})$ & 250 & 500 \\
\hline Repetition rate $f \mathrm{~N}_{\text {bunch }}(\mathrm{Hz})$ & $5 * 2625$ & $4 * 2625$ \\
\hline Number of particles/bunch & $2.0510^{10}$ & $2.0510^{10}$ \\
\hline $\begin{array}{l}\text { Normalized emittances } \gamma \varepsilon_{\mathrm{x}} / \gamma \varepsilon_{\mathrm{y}} \\
(\mathrm{m} . \mathrm{rad})\end{array}$ & $110^{-5} /$ & $110^{-5} /$ \\
$410^{-8}$ & $410^{-8}$ \\
\hline Relativistic factor $\gamma$ & 833.912 & 1667.82 \\
\hline IP Beta functions $\beta_{\mathrm{x}} / \beta_{\mathrm{y}}(\mathrm{mm})$ & $20 / 0.4$ & $30 / 0.3$ \\
\hline IP Beam sizes $\sigma_{\mathrm{x}} / \sigma_{\mathrm{y}}(\mathrm{nm})$ & $639.4 / 5.7$ & $553.7 / 3.5$ \\
\hline $\begin{array}{l}\text { Geometric luminosity } \mathrm{L}_{0} \\
\left(\mathrm{~cm}^{-2} . \mathrm{s}^{-1}\right)\end{array}$ & $1.2010^{34}$ & $1.8110^{34}$ \\
\hline
\end{tabular}

*Work supported by the EC under the FP6 "Research Infrasctructure Action - Structuring the European Research Area" EUROTeV DS Project Contract no.011899 RIDS

\#payet@cea.fr

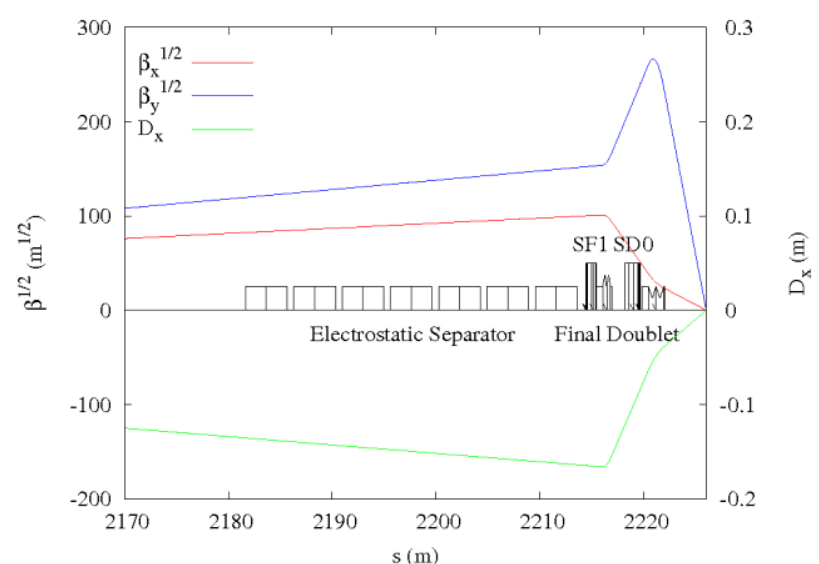

Figure 1: FD region optical functions at $500 \mathrm{GeV}$.

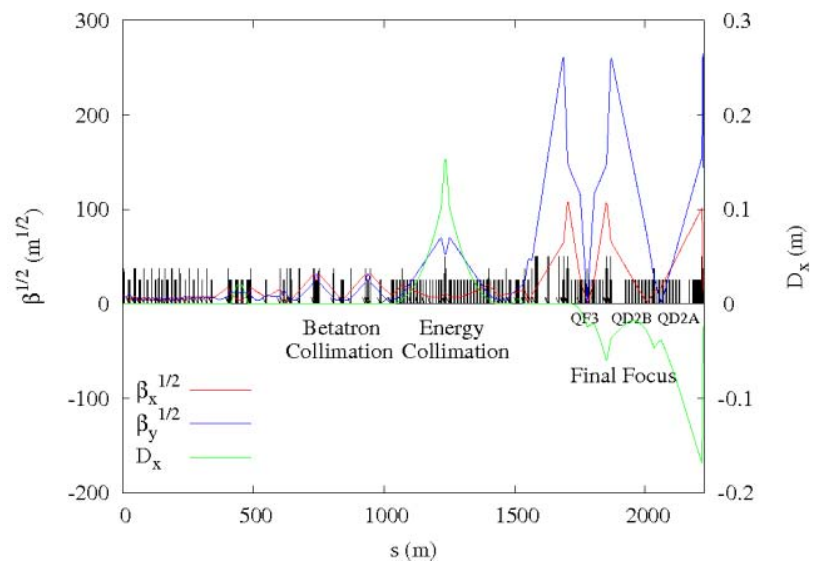

Figure 2: BDS optical functions at $500 \mathrm{GeV}$.

\section{LUMINOSITY OPTIMISATION PROCEDURES}

The optimization procedure has to minimize, at the IP, the product $\Sigma_{I P}=\overline{(x-\bar{x})^{2}} \cdot \overline{(y-\bar{y})^{2}}$. Two approaches are used:

- We use analytical expressions of the rms beam sizes based on the beam distribution parameters and the high order transfer matrix terms. So, we develop the code LUMOPT [8] which computes analytically the beam sizes and minimizes their product.

- We use the code TRACEWIN which tracks a particle cloud to the IP, computes the rms dimensions and searches for a minimum of the product.

Both codes search for a minimum by varying 14 elements (8 quadrupoles, and 6 sextupoles) of the BDS. The beam momentum spread is an optimisation 
parameter. If needed, the beta functions at IP are matched to the nominal ones after the optimisation.

\section{Analytical Expression of rms beam sizes}

In order to express the rms value of the transverse beam sizes at the IP as a function of the $2^{\text {nd }}$ and $3^{\text {rd }}$ order matrix terms, we write the expression of the particle co-ordinates transport:

$x_{i}=\sum_{j} R_{i j} x_{j}^{(0)}+\sum_{j k} T_{i j k} x_{j}^{(0)} x_{k}^{(0)}+\sum_{j k l} U_{j k l} x_{j}^{(0)} x_{k}^{(0)} x_{l}^{(0)}+o(3)$

Where $x_{i}^{(0)}$ is the value of the $\mathrm{i}^{\text {th }}$ particle co-ordinate at the transport line entry, $R_{i j}, T_{i j k}, U_{i j k l}$ are respectively the $1^{\text {st }}, 2^{\text {nd }}$, and $3^{\text {rd }}$ order terms of the transfer matrix.

We assume that the beam distribution at the beam line entry is a 6D Gaussian, then, all the odd moments of the beam distribution are equal to zero.

The mean values of the co-ordinates are then:

$$
\overline{x_{i}}=\sum_{j k} T_{i j k} \overline{x_{j}^{(0)} x_{k}^{(0)}}
$$

One can write the $4^{\text {th }}$ order moments as functions of the $2^{\text {nd }}$ moments [9]:

$\overline{x_{j}^{(0)} x_{k}^{(0)} x_{l}^{(0)} x_{m}^{(0)}}=\overline{x_{j}^{(0)} x_{k}^{(0)}} \cdot \overline{x_{l}^{(0)} x_{m}^{(0)}}+\overline{x_{j}^{(0)} x_{l}^{(0)}} \cdot \overline{x_{k}^{(0)} x_{m}^{(0)}}$

$+\overline{x_{j}^{(0)} x_{m}^{(0)}} \cdot \overline{x_{k}^{(0)} x_{l}^{(0)}}$

The $6^{\text {th }}$ order beam moments can be expressed by the same way. So we can write the standard deviation as:

$$
\begin{aligned}
& \overline{\left(x_{i}-\overline{x_{i}}\right)^{2}}=\sum_{j j^{\prime}} R_{i j} R_{i j^{\prime}} \overline{x_{j}^{(0)} x_{j^{\prime}}^{(0)}} \\
& +\sum_{j k} \sum_{j^{\prime} k^{\prime}} T_{i j k} T_{i j^{\prime} k^{\prime}}\left(\begin{array}{l}
\overline{x_{j}^{(0)} x_{k}^{(0)} x_{j^{\prime}}^{(0)} x_{k^{\prime}}^{(0)}} \\
-\overline{x_{j}^{(0)} x_{k}^{(0)}} \cdot \overline{x_{j^{\prime}}^{(0)} x_{k^{\prime}}^{(0)}}
\end{array}\right) \\
& +2 \sum_{j} \sum_{j^{\prime} k^{\prime} l^{\prime}} R_{i j} U_{i j^{\prime} k^{\prime} l^{\prime}} \overline{x_{j}^{(0)} x_{j^{\prime}}^{(0)} x_{k^{\prime}}^{(0)} x_{l^{\prime}}^{(0)}} \\
& +\sum_{j k l} \sum_{j^{\prime} k^{\prime} l^{\prime}} U_{i j k l} U_{i j^{\prime} k^{\prime} l^{\prime}} \overline{x_{j}^{(0)} x_{k}^{(0)} x_{l}^{(0)} x_{j^{\prime}}^{(0)} x_{k^{\prime}}^{(0)} x_{l^{\prime}}^{(0)}}
\end{aligned}
$$

The matrix terms $R, T, U$ are given by TRANSPORT.

To keep the $U_{i j k l} U_{i j^{\prime} k^{\prime} l^{\prime}}$ terms, mathematically we should have to develop the co-ordinates up to the 5th order. Two contributions are missing, the $T_{i j k} V_{i j^{\prime} k^{\prime} l^{\prime} n^{\prime}}$ and $R_{i j} W_{i j^{\prime} k^{\prime} l^{\prime} n^{\prime} m^{\prime}}$ terms, where $V_{i j^{\prime} k^{\prime} l^{\prime} n^{\prime}}$ and $W_{i j^{\prime} k^{\prime} l^{\prime} n^{\prime} m^{\prime}}$ are respectively the 4 th order and 5 th order terms of the transfer matrix. But without the $U_{i j k l} U_{i j^{\prime} k^{\prime} l^{\prime}}$ term contribution the optimisation is less efficient. In all cases, that beam sizes representation gives only an estimate of the rms beam sizes [9].

\section{LUMINOSITY COMPUTATION}

To compare the luminosity resulting from different optimisation processes, we always use the same procedure. We track 6D Gaussian distribution particle clouds, truncated to $3 \mathrm{rms}$ in $x, x^{\prime}, y, y^{\prime}, l$ and to $2 \mathrm{rms}$ in momentum with DIMAD [10], by varying the beam rms momentum spread $\sigma_{d p / p}$.

For each momentum spread, we count at the IP the particles which are in an elementary cell, $\mathrm{n}_{\mathrm{i}}$, of a $60 \sigma_{x}^{(I P)} \times 120 \sigma_{y}^{(I P)}$ rectangular surface, where $\sigma_{x}^{(I P)}$ and

$\sigma_{y}^{(I P)}$ are respectively the horizontal and vertical nominal beam sizes at IP, centred on the machine axis and divided by a $240 \times 480$ grid. The luminosity is computed by integrating the overlap of the transverse beam distribution on itself [11].

\section{RESULTS}

\section{GeV Beam Line Optimisation}

Figure 3 shows the optimised luminosity as a function of the beam momentum spread of the $500 \mathrm{GeV}$ BDS for 3 values of the beam momentum spread optimisation parameter. As one can see, in the 3 cases, the reached luminosity curves are close to the expected flat curve even if the initial luminosity curve shape is different from the objective. One can remark also that for the large values of the momentum spread parameter the luminosities are better at large scale but can be lower near the origin.

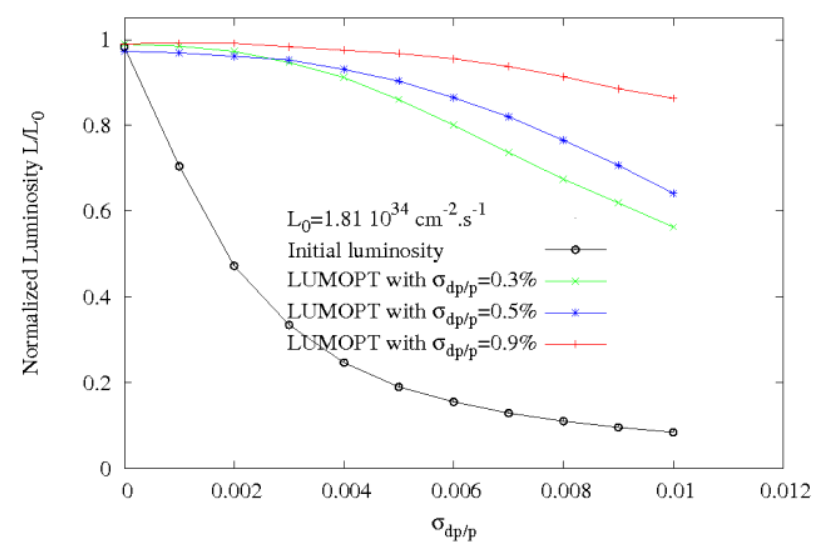

Figure 3: LUMOPT optimisations at $500 \mathrm{GeV}$.

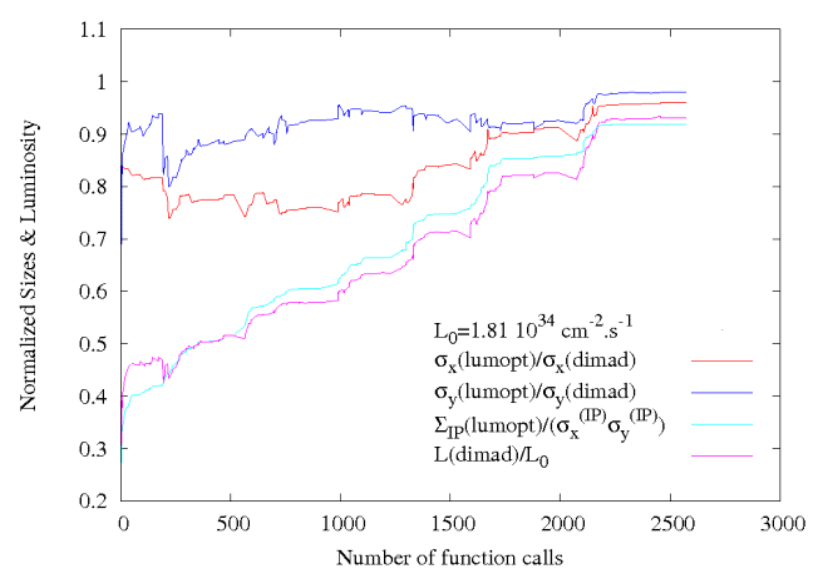

Figure 4: Evolution during LUMOPT optimisation with $\sigma_{\mathrm{dp} / \mathrm{p}}=0.5 \%$. 
The evolution during the optimisation process of the analytic beam sizes, normalised by the rms beam sizes obtained by particles tracking with DIMAD, and the normalised luminosities are plotted on Figure 4. The agreement between the analytic beam size computation and the particle cloud tracking becomes better at the end of the processes, since the non-linearities are reduced. The luminosity from particle cloud tracking does not always grow. The decreases are due to the limits of the formulas representation. But despite this, the evolution of the curve follows the evolution of the merit value.

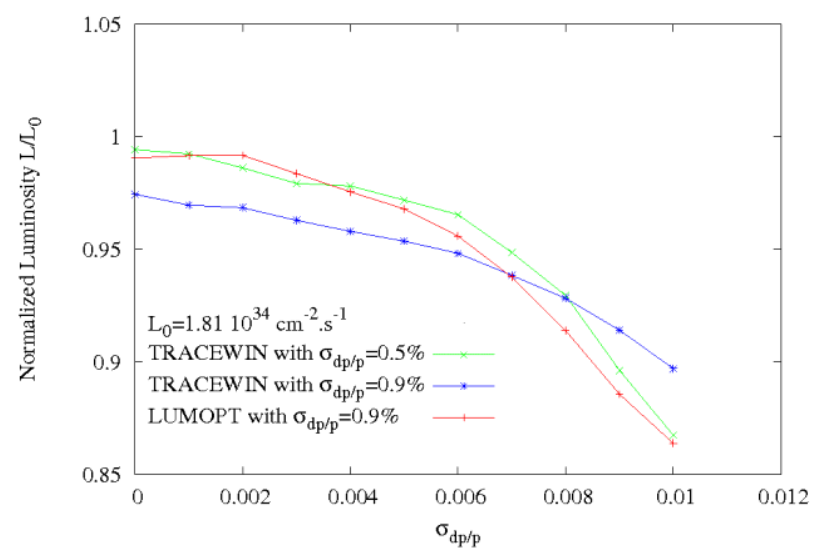

Figure 5: Comparison between TRACEWIN and LUMOPT optimisations at $500 \mathrm{GeV}$.

The Figure 5 shows the comparison between the LUMOPT and TRACEWIN optimisations. The two codes give similar results and have the same behaviour toward the momentum spread optimisation parameter.

\section{GeV Beam Line Optimisation}

Figure 6 shows the optimised luminosity of the $250 \mathrm{GeV}$ BDS for 3 values of the beam momentum spread parameter. The starting point is close to the final state and the code LUMOPT obtains best results than $L_{0}$ up to $0.3 \%$ due to a dense core beam for the final distribution.

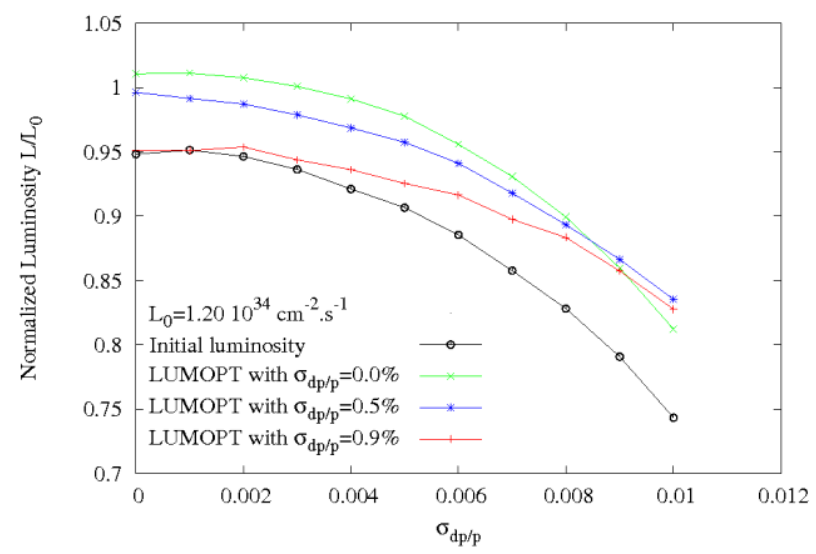

Figure 6: LUMOPT optimisation at $250 \mathrm{GeV}$.

Figure 7 shows the luminosity of a $250 \mathrm{GeV}$ BDS with $l *=6 \mathrm{~m}$. In this case, the luminosity decreases more quickly at large momentum, but it remains larger than $0.88 \mathrm{~L}_{0}$ up to $0.5 \%$.

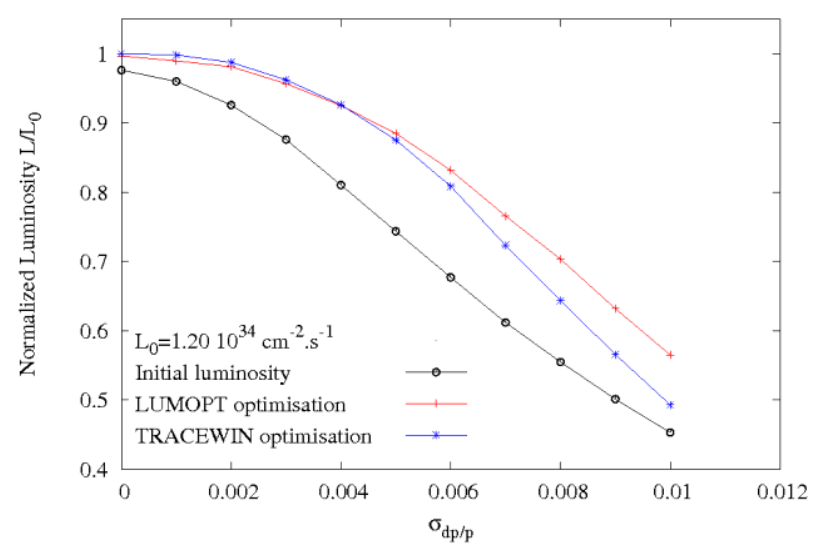

Figure 7: Optimisation for a $250 \mathrm{GeV}$ BDS with $l^{*}=6 \mathrm{~m}$.

\section{CONCLUSIONS}

Both codes LUMOPT and TRACEWIN allow the automatic optimisation of the BDS luminosity. The obtained luminosity curves are comparable to the $14 \mathrm{mrad}$ and 2 mrad BDS one. The code LUMOPT reaches its goal in a relatively short time in comparison with the time needed by the tracking code TRACEWIN. But, the results depend on the starting point, so we are studying improvements about the optimisation algorithm, the merit function, and the initial lattice tuning in order to facilitate the optimisation.

\section{REFERENCES}

[1] P. Raimondi and A. Seryi, "A novel final focus design for future linear colliders", Phys. Rev. Lett. 86, 3779 (2001).

[2] R.Tomás, "Non-linear Optimization of Beam Lines", CERN - AB Division, CLIC Note 659.

[3] K.L. Brown, D.C. Carey, Ch. Iselin, and F. Rothacker, "TRANSPORT A Computer Program for Designing Charged Particle Beam Transport Systems," SLAC-91 (1973 Rev.), FNAL-91, and CERN 80-04.

[4] R. Duperrier, N. Pichoff, D. Uriot, CEA Saclay codes review, ICCS Conference, Amsterdam 2002.

[5] L. Keller, "Zero Degree Extraction using an Electrostatic Separator", ILC Snowmass, August 2005.

[6] J. Borburg, "ILC Separator proposal", Daresbury Meeting, February $26 / 27^{\text {th }}, 2007$.

[7] M. Woodley, "ILC Beam Delivery System Hybrid Layout 2006e Release", SLAC BDS Group, October 31, 2006.

[8] S. Auclair, "Rapport de stage: création d'un programme d'optimisation automatique", EFREI, September 2006.

[9] D. Carey, "The Optics of Charged Particle Beams", harwood academic publishers, p204.

[10] R V. Servranckx, K L. Brown, L. Schachinger, D. Douglas, "Users Guide to the Program DIMAD", SLAC REPORT 285 UC-28 (A), May 1985

[11] O. Napoly, "The luminosity for beam distributions with errors and wakefield effects in linear colliders", Particle Accelerators 40/4, p 181-203, 1993. 